\title{
Review on Facial Recognition
}

\author{
Mubashshir $^{1}$, Dr. Kshitij Shinghal ${ }^{2}$, Mr. Manas Singhal $^{3}$ \\ ${ }^{1,2,3}$ Department of Electronics and Communication Engineering \\ ${ }^{1,2,3}$ Moradabad Institute of Technology, Moradabad.
}

\begin{abstract}
Generally face recognition perform many operations in our daily life such as security purpose identification of people and verification purpose. The basic aim of my project is to design an effective and secure technique for authentication using face recognition that can search or recognize a human face among the thousands of persons and improve the performance of face recognition system in low light conditions and also evaluate the performance of the designed framework by comparing the performance of existing face recognition system. This study also provides a automatic system through which a given still image or video of a scene, identify one or more persons in this scene by using a stored database of facial images.
\end{abstract}

Keywords: Face Recognition, Face Identification, Biometric, Face Authentication, Find Eigen Face, Preparing Dataset. $* * * * *$

\section{Introduction}

Face recognition it is used for the identification of a person or you can say recognition second a this system are use for the verification of a person or authentication purpose and facial recognition systems this are commonly use for the security purpose for verification and identification. Next purpose is the can be track to journey so different facial features are trackt at the airport. Image and vision processing is a part of face recognition. This vision processing there are three field such as military, biometric, security. I working on biometric in the biometric again it is further classified in to fingerprint recognition, hand geometry recognition, facial recognition, voice recognition, signature recognition. So I choose face recognition. Biometric is a technology which is used for the identification, and analyze the persons and to measure and to measure an individuals. The biometrics divided into two part first is bahavioral biometrics (based on measurements and data derived from an action) such as- voice-scan, signaturescan, keystroke-scan second is physiological biometrics (based on measurements and data derived from direct measurement of a part of the human body) such as- fingerscan, facial recognition, iris recognition retina-scan, handscan.

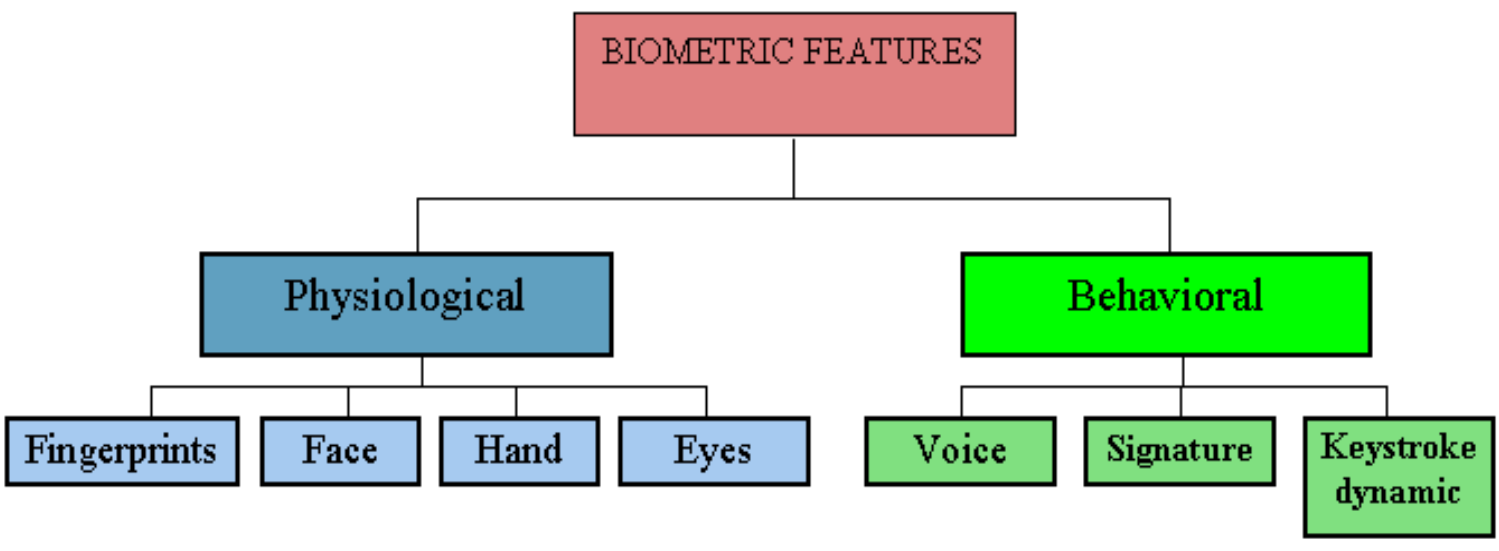

Fig.1.1 Classification of Biometrics Features

\subsection{Why face Recognition}

A person how we can easily identified person. Why face because face is unique part of human body and through face we can easily identified any one. A person can also be easily by his voice but voice is an aggriconcept next is how computer can recognize a human from digital image and from video frame. So further face recognition idon't need one to give me fingerprint. I need you voice sample or anything. I just need your digital image and that can identify you and next is face recognition technology analyze the unique shape, pattern an positioning of facial feature. 


\subsection{Objectives}

The objectives of low light image denoising for facial recognition system are as follows:

1. To study and analysis the existing work in face recognition.

2. To develop a more effective algorithm than existing algorithms to make the

face recognition accurately.

3. Performance Evaluation of proposed framework by comparing performance of existing facial recognition system.

4. Design of a security sample to protect face recognition system.

5. Classify the test image acc to the training and calculate performance- Recognition rate, Visual quality of image, Time complexity.

\section{Literature Review}

Yimei Kang and Wang Pan et al. In this paper author mentioned that illumination variation makes face recognition a tough and difficult task in a very low light atmosphere. During this paper author proposed a very easy and economical novel approach of low light image denoising of low frequency noise is termed DeLFN and also the noise distribution of low light pictures is represented supported large experimental results. In low light condition the low frequency and extremely low frequency noise element are dominant. The DeLFN technique is 3 level low light image denoising technique for face recognition. The primary level of DeLFN methodology, it denoises mixes noises using bar graph equalization technique and therefore improve overall contrast of pictures. The second level delfn methodology work on a low frequency noise element to enhance the image details by the logarithmic transformation (LOG) technique. And also the third level denoises remaining very low frequency noise elements that are present on the image through the high pass filter, this method recovers a lot of options of actuality image. Next PCA ( Principal component Analysis) recognition technique is applied to examine recognition rate of the pre-processed face image of DeLFN approach. The author compared DeLFN a technique with many representatives preprocessing strategies. In DeLFN methodology not only outperformed different algorithms and important the rising scenes quality and face recognition rate for image, however this method additionally easy for real time application.

Ognjen Arandjelovic' a,*, Roberto Cipolla et al. In this paper to realize illumination invariableness within the presence of enormous cause changes remains one among automatic face recognition. Author said that the strategy relies on outputs of easy image process filters that contend with unprocessed grayscale input of an easy matching score between two people. He shows distinction of the illumination conditions between question input and coaching ( gallery) dataset will be estimated and wont to weight the contributions of two competitor representations. This illustration of weight parameters is particular ( i.e filter specific) however not gallery specific. So computationally demanding, learning stage algorithm is offline based mostly and desires to be performed solely, creating supplementary on-line overhead lowest. Finally he describes intensive empirical analysis of the projected technique in each a video and still image-based setup is performed five databases. This databases total 333 people, over 1660 video sequence and 650 still pictures, and extreme variation in illumination pose, head motion, etc. In this difficult dataset algorithm demonstrate a performance improvement over traditional filtering approaches. They demonstrate a discount of 50$70 \%$, in recognition rates. The simplest activity filter technique is combination properly recognizing in ninety seven of people.

Shruti Nagpal, Maneet Singh, Richa Singh, And Mayank Vatsa et al. In this paper author has discussed about the importance of body weight to recognize the human face. They said that integral variation of body weight in aging process has changed features of a human face. And he also said that the lack in proper relationship between the age and weight makes difficult to recognize the human face. Author proposed regularizer based approach to learn weight in- variant facial representation using two different architectures namely, sparse-stacked denoising auto encoders and deep Boltzmann machines. And author also said that several experiments performed on the extended wit database show that use of wait aware regularization improves the face recognition accuracy without any dropout.

June-Young Jung, Seung-Wook Kim, CheolHwan Yoo, Won-Jae Park, And Sung-Jea Ko, Fellow et al. In this paper author considered that most of the facial recognition (FR) system at the present time initially identifies the discriminative feature from a facial image available in the database and then classify them based on different features. Author proposes a method to identify these human facial traits and a low-dimensional features extraction method using OLDA. The proposed features relies on a local binary pattern to represent texture information and random ferns to build a structural model. By identifying and integrating these feature vectors from OLDA method, it achieves a high dimension of the input facial image. In general way, the discriminative ability of a FR system is depends upon the feature dimension of the input facial image. However, it is very difficult to compute higher dimension of facial image as it makes the whole process more complex and prone to mistakes. So it is concluded that dimensionality must be reduced to eliminate 
these mistakes in practical FR application. OLDA is a technique, utilized to scale back the size of the known features and improve its discriminative ability with representative FR information, the proposed technique demonstrates a better recognition rate and low procedure quality compared to existing FR strategies. Additionally to a facial image database disguises, the projected technique demonstrates outstanding performance.

Hongwen Zhang, Qi Li, Zhenan Sun, Member, IEEE, and Yunfan Liu et al. In this Paper the Author discussed regarding Facial mark feature Detection of human faces. The Facial mark feature Detection Is a crucial, however also a tough task to perform for world Application of face detection. During this paper the author must be thought of a strong combining data driven technique for facial landmark detection. The primary step is to use a fullytrained convolutional network (FCN) to see a response of all facial landmark points. This data driven technique will totally use this holistic information in an exceedingly facial image for international estimation of facial landmark. Currently within the next step, a pre trained purpose distribution model (PDM) generate an initial facial shape from the maximum point response of FCN. This model driven approach is ready to correct the wrong location of outliers in facial image. Finally, verify the knowledge and once the think about form for facial pictures. At last, a regularised landmark mean-shift (RLMS) technique is to use to fine tune the facial form of the image. The author aforesaid, that this estimated technique uses to the correct calibration method and absolutely combines the worldwide robustness benefits from the data driven technique (FCN). During this experiment the author must be thought of the perform to achieve four datasets is available like $300 \mathrm{~W}$, $\mathrm{AFLW}, \mathrm{AFW}$, and COFW. The projected technique is ready to provide a satisfying detection results face pictures with expression, massive head poses etc.

Haoxi Li, Student Member, IEEE, Haifeng Hu , Member, IEEE, and Chitung Yip et al. In this paper author discuss about a deep convolutional network method for cross-age face recognition called age related factor guided joint task modeling convolutional neural network.
Author said that cross-age face recognition has remained a popular have failed in dealing with facial changes through age, hence to improve the cross-age face recognition and to reduce the errors he proposed a system that combines an identity discrimination network with an age discrimination network that shares the same feature layers. By as an alternative coaching, the cross-age identity options and cross-identity, age options will be separated with high-inter category distension and intra-class compactness. And this process has been performed three benchmark face aging datasets ( MORPH Album 2, CACD-VS, CALW ). The results of our model shows more effective and superior performance.

Hongjun Wang, Jiani Hu, And Weihong Deng et al. Represented robust face recognition at the encoding of facial descriptors among numerous encoders and fisher vector technique. During this paper the author must be considered FV methodology in an exceedingly new manner, first they propose a new CFV descriptor. The CFV is obtained by zeroing little posteriors, calculative first-order statistics and reweighting component properly. The second methodology is light of iterative quantization (ITQ) scheme. They present a generalized ITQ methodology, this GITQ scheme will facilitate the binarize our CFV. Finally, They apply CFV and GITQ scheme to encode convolutional activations from convolutional neural networks. They evaluate our methodology four kinds of dataset names like FERET, LFW, AR, and our experiment tested the advantage of this methodology.

Dayong Wang et al. Represented Face Search at the scale. In this paper author proposed a cascade search system appropriate for large-scale face search issues. Because of the popularity of social media websites, one challenge facing computer vision researchers is to devise technique search for a person of interest among the billions of shared photos on these websites. Searching an oversized collection of unconstrained face pictures remains a difficult drawback. Author projected a face search system coupled with industrial off the shelf matchmaker.

Table 1: Comparison of Different Research Paper for Facial Recognition

\begin{tabular}{|c|c|c|c|c|c|}
\hline S.N. & Authors & Title and publishers & Strength, Algorithm & Weakness & Opportunities \\
\hline 1. & 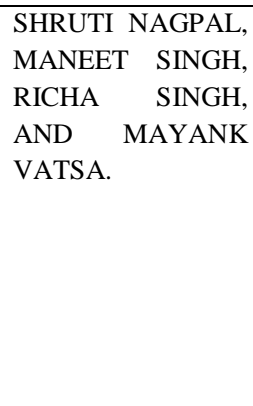 & $\begin{array}{l}\text { Regularized Deep Learning } \\
\text { for Face Recognition With } \\
\text { Weight Variations. } \\
\text { December 2015, IEEE } \\
\text { ACCESS. }\end{array}$ & $\begin{array}{l}\text { Strength:- } \\
\text { (1) Improves the identification } \\
\text { Accuracy of the Architecture } \\
\text { Without Dropout. } \\
\text { (2) Increase in the number of } \\
\text { images in the gallery set }[10] \text {. } \\
\text { (3) Deep learning based frame } \\
\text { Work. } \\
\text { Algorithm:- }\end{array}$ & $\begin{array}{l}\text { (1) Due to the complex } \\
\text { Architecture. } \\
\text { (2) Low generalizat- } \\
\text { ion power and poor } \\
\text { Prediction Results. }\end{array}$ & $\begin{array}{l}\text { This research can be } \\
\text { further extended in } \\
\text { multiple directions: } \\
\text { extend the database to } \\
\text { include more images } \\
\text { with weight variations, } \\
\text { and improve the } \\
\text { algorithm with } \\
\text { incorporating both age } \\
\text { and weight variations. }\end{array}$ \\
\hline
\end{tabular}




\begin{tabular}{|c|c|c|c|c|c|}
\hline & & & $\begin{array}{l}\text { (1) Boltzman Machines(DBM). } \\
\text { (2)Restricted Boltzmann Machines } \\
\text { (RBM). } \\
\text { (3)SDAE, Commercial-Off-The- } \\
\text { Shelf (COTS). }\end{array}$ & & \\
\hline 2. & $\begin{array}{l}\text { HONGWEN } \\
\text { ZHANG, QI LI, } \\
\text { ZHENAN SUN, } \\
\text { MEMBER, IEEE, } \\
\text { AND YUNFAN } \\
\text { LIU. }\end{array}$ & $\begin{array}{l}\text { Combining Data-Driven } \\
\text { And Model-Driven Methods } \\
\text { For Robust Facial } \\
\text { Landmark Detection. } \\
\text { October 2018, } \\
\text { IEEE TRANSACTION. }\end{array}$ & $\begin{array}{l}\text { Strength:- } \\
\text { (1) Outlier correction capability of } \\
\text { the facial images. } \\
\text { (2) Able to produce satisfying } \\
\text { detection results } \\
\text { on face images. } \\
\text { (3) ECT method achieves superior, } \\
\text { or at least comparable } \\
\text { performance. } \\
\text { Algorithm:- } \\
\text { (1) ECT (estimation-correction- } \\
\text { tuning) for facial landmark } \\
\text { detection. }\end{array}$ & $\begin{array}{l}\text { (1) Effectiveness on } \\
\text { face images with } \\
\text { extreme appearance } \\
\text { variations, large head } \\
\text { poses and heavy } \\
\text { occlusions. } \\
\text { (2) In computer vision } \\
\text { and pattern recognition } \\
\text { problems. } \\
\text { (3)Time Complexity. }\end{array}$ & $\begin{array}{l}\text { This research can be } \\
\text { ECT further extended of } \\
\text { general object alignment } \\
\text { of human pose } \\
\text { estimation and image } \\
\text { segmentation etc. }\end{array}$ \\
\hline 3. & $\begin{array}{lr}\text { YIMEI } & \text { KANG } \\
\text { AND WANG PAN. }\end{array}$ & $\begin{array}{l}\text { A Novel Approach Of Low- } \\
\text { Light Image Denoising For } \\
\text { Face Recognition. } \\
\text { Hindawi Publishing } \\
\text { Corporation Advances in } \\
\text { Mechanical Engineering, } \\
\text { APRIL } 2014\end{array}$ & $\begin{array}{l}\text { Strength:- } \\
\text { (1) DeLFN Contains More } \\
\text { Essential Information For Face } \\
\text { Recognition. } \\
\text { (2) Greatly reduces the } \\
\text { illumination changes. } \\
\text { (3) Image quality for visual } \\
\text { effects and various illumination } \\
\text { conditions and it is very stable } \\
\text { violent. } \\
\text { Algorithm:- } \\
\text { (1) The DeLFN Algorithm. }\end{array}$ & $\begin{array}{l}\text { (1) Low-light Noise is a } \\
\text { Significant Problem in } \\
\text { Robotic Automatic Face } \\
\text { Recognition. } \\
\text { (2) In Low-light } \\
\text { Environments, The } \\
\text { Performance of Face } \\
\text { Recognition } \\
\text { Degrades. }\end{array}$ & $\begin{array}{l}\text { Low-light noise is a } \\
\text { significant problem in } \\
\text { robotic automatic face } \\
\text { recognition, this is one } \\
\text { possible future direction } \\
\text { for this study. }\end{array}$ \\
\hline 4. & $\begin{array}{l}\text { HAOXI LI, } \\
\text { STUDENT } \\
\text { MEMBER, IEEE, } \\
\text { HAIFENG HU, } \\
\text { MEMBER, IEEE, } \\
\text { AND CHITUNG } \\
\text { YIP. }\end{array}$ & $\begin{array}{l}\text { Age-Related factor or } \\
\text { guided joint Task modeling } \\
\text { Convolution neural network } \\
\text { For cross-age face } \\
\text { Recognition. } \\
\text { SEPTEMBER 2018, } \\
\text { IEEE } \\
\text { TRANSACTION. }\end{array}$ & $\begin{array}{l}\text { Strength:- } \\
\text { (1) Enhance the system capability } \\
\text { of face recognition. } \\
\text { (2) Cross-age face recognition has } \\
\text { attracted research and sufficiently } \\
\text { reliable solution [11]. } \\
\text { (3) Greatly boosting the face } \\
\text { recognition accuracy. } \\
\text { Algorithm:- } \\
\text { (1) Model Training for JTFA. } \\
\text { (2) Model Training for AFJT- } \\
\text { CNN. }\end{array}$ & $\begin{array}{l}\text { (1) These method fail to } \\
\text { achieve a more } \\
\text { satisfactory result due to } \\
\text { the inherent defects of } \\
\text { the traditional } \\
\text { approaches. } \\
\text { (2) The main } \\
\text { disadvantage of this } \\
\text { model it requires a large } \\
\text { number of training } \\
\text { samples. }\end{array}$ & $\begin{array}{l}\text { This research can be } \\
\text { Further extended new } \\
\text { deep learning network } \\
\text { architecture called } \\
\text { AFJT-CNN } \\
\text { Specifically address the } \\
\text { cross age-face } \\
\text { recognition task. }\end{array}$ \\
\hline
\end{tabular}




\begin{tabular}{|c|c|c|c|c|c|}
\hline 5. & $\begin{array}{l}\text { HONGJUN WANG, } \\
\text { JIANI HU, AND } \\
\text { WEIHONG DENG. }\end{array}$ & $\begin{array}{l}\text { Compressing Fisher Vector } \\
\text { for Robust face recognition. } \\
\text { November } 2017 \text {, } \\
\text { IEEE ACCESS. }\end{array}$ & $\begin{array}{l}\text { Strength:- } \\
\text { (1) CNNs enhance traditional } \\
\text { features by providing the trade-off } \\
\text { between 'breadth' and 'depth'. } \\
\text { (2) CFV to achieve better accuracy. } \\
\text { (3) Provide a flexible hashing } \\
\text { method called GITQ. } \\
\text { Algorithm:- } \\
\text { (1) GITQ hashing algorithm } \\
\text { ( ITQ). } \\
\text { (2) CFV encoding algorithm [43]. } \\
\text { (3) SP and LSH, Near-optimal } \\
\text { hashing algorithms [14]. }\end{array}$ & $\begin{array}{l}\text { (1) The issue of } \\
\text { compressing FV and } \\
\text { retaining at the same } \\
\text { time. } \\
\text { (2) Time consuming } \\
\text { problem. } \\
\text { (3) The problem of } \\
\text { large-scale image } \\
\text { search, binarization } \\
\text { [6] and product } \\
\text { quantization [5]. }\end{array}$ & $\begin{array}{l}\text { This research can be } \\
\text { Further extended to } \\
\text { encode deep convolu- } \\
\text { tional activations. The } \\
\text { approaches shows pr- } \\
\text { mosing results for face } \\
\text { recogination. }\end{array}$ \\
\hline 6. & \begin{tabular}{lr}
\multicolumn{2}{c}{ JUNE-YOUNG } \\
JUNG, SEUNG- \\
WOOK & KIM, \\
CHEOL-HWAN \\
YOO, WON-JAE \\
PARK, & AND \\
SUNG-JEA & KO, \\
FELLOW.
\end{tabular} & $\begin{array}{l}\text { LBP-Ferns-Based Feature } \\
\text { Extraction for Robust Facial } \\
\text { Recognition. } \\
\text { November 2016, } \\
\text { IEEE } \\
\text { TRANSACTION. }\end{array}$ & $\begin{array}{l}\text { Strength:- } \\
\text { (1) OLDA method improves the } \\
\text { accuracy of FR system. } \\
\text { (2) OLDA shows better rates as } \\
\text { compared with LDA method. } \\
\text { (3) The Computation time of the } \\
\text { proposed method was fastest. }\end{array}$ & $\begin{array}{l}\text { (1) Under sampled } \\
\text { problem for FR system. } \\
\text { (2) The FR System } \\
\text { difficulty of the LFW } \\
\text { database is } \\
\text { Comparatively hig-her } \\
\text { than the other databases. }\end{array}$ & $\begin{array}{l}\text { The main direction for } \\
\text { future work is to } \\
\text { develop reduction of } \\
\text { computational compl- } \\
\text { exity is important to } \\
\text { extend the usage time of } \\
\text { practical devices. }\end{array}$ \\
\hline 7. & $\begin{array}{l}\text { Ognjen arandjelovic } \\
a,{ }^{*} \text {, roberto cipolla }\end{array}$ & $\begin{array}{l}\text { A methodology for rapid } \\
\text { illumin-tion invariant face } \\
\text { recognition using image } \\
\text { processing filter. } \\
\text { February 2009, } \\
\text { Elsevier. }\end{array}$ & $\begin{array}{l}\text { Strength:- } \\
\text { (1) Reducing the amount of offline } \\
\text { training data. } \\
\text { (2) Reducing the avg recognition } \\
\text { error rate. } \\
\text { (3) Simple image formation } \\
\text { models. } \\
\text { Algorithm:- } \\
\text { (1) Offline training algorithm. }\end{array}$ & $\begin{array}{l}\text { 1) By formulating the } \\
\text { problem of estimating } \\
\text { this similarity in a } \\
\text { discriminative manner. } \\
\text { (2) A problem robust } \\
\text { image filter that can } \\
\text { deal with more complex } \\
\text { illumination effects. } \\
\text { (3) A main problem } \\
\text { causing greater practical } \\
\text { difficulties than varying } \\
\text { pose. }\end{array}$ & $\begin{array}{l}\text { The main direction for } \\
\text { future work is to } \\
\text { develop more robust } \\
\text { image processing filter } \\
\text { without a great loss of } \\
\text { computational } \\
\text { efficiency. }\end{array}$ \\
\hline
\end{tabular}

\section{Conclusion}

Face recognition may be a part of a pattern recognition technology recognition, especially covers a variety of activities from several walks of life. The nowadays core of technology ready to mechanically verify determine data for secure transactions, for police work and security tasks for access management to buildings etc. This application sometimes works in controlled environments and recognition algorithms will take get high recognition accuracy. As a result, there aren't any technological or money barriers, pilot program or widespread readying and it'll acknowledge the face or will avoid some unwanted terrorist without touch the persons. For implementations wherever the biometric system should verify and determine users dependably over time, facial scan can be a very difficult, but not impossible, this technology to implement successfully.

\section{Acknowledgment}

I sincerely want to thank our guide, Dr. Kshitij Shinghal (Associate Professor), Mr. Manas Singhal (Assistant Professor) the department of Electronics and Communication Engineering, for giving us his precious time and guidance in completing this survey paper. In the completion of this paper, there is a significant role of our teacher, Mr. Manas Singhal Sir. For department of Electronics and Communication Engineering. His valuable advice and motivation keep us going and results in the completion of this paper.

\section{References}

[1] Swati S. Bobde, " Face Recognition Technology ", International Journal of Computer Science and Mobile Computing, VOL. 03, pp. 192-202, October 2014.

[2] Yimei Kang and Wang Pan," A Novel Approach of LowLight Image Denoising for Face Recognition", Hindawi Publishing Corporation Advances in Mechanical Engineering, VOL. 2014, pp. 01-13, April 2014. 
[3] Ognjen Arandjelovic' a, *, Roberto Cipolla,' A methodology for rapid illumination-invariant face recognition using image processing filters", Elsevier, VOL. 113, pp. 159-171, February 2009.

[4] SHRUTI NAGPAL, MANEET SINGH, RICHA SINGH, AND MAYANK VATSA,' Regularized Deep Learning for Face Recognition With Weight Variations', IEEE TRANSACTIONS Special Section On Applying Four Ds Of Machine Learning To Advance Biometrics, VOL.03, pp. 3010-3018, December 2015.

[5] JUNE-YOUNG JUNG, SEUNG-WOOK KIM, CHEOLHWAN YOO, WON-JAE PARK, AND SUNG-JEA KO, FELLOW, " LBP-Ferns-Based Feature Extraction for Robust Facial Recognition", IEEE Transactions on Consumer Electronics, VOL. 62, NO. 04 pp. 446-453, November 2016.

[6] Hongwen Zhang, Qi Li, Zhenan Sun, Member, IEEE, and Yunfan Liu,' Combining Data-Driven and Model-Driven Methods for Robust Facial Landmark Detection', IEEE TRANSACTIONS ON INFORMATION FORENSICS AND SECURITY, VOL. 13, NO. 10, OCTOBER 2018.

[7] Haoxi Li, Student Member, IEEE, Haifeng Hu , Member, IEEE, and Chitung Yip,' Age-Related Factor Guided Joint Task Modeling Convolutional Neural Network for CrossAge Face Recognition", IEEE TRANSACTIONS ON INFORMATION FORENSICS AND SECURITY, VOL. 13, NO. 09, pp. 2381-2392, September 2018.

[8] HONGJUN WANG, JIANI HU, AND WEIHONG DENG,", Compressing Fisher Vector for Robust Face Recognition', IEEE ACCESS Publishing, VOL.05, pp. 23157-23165, November 2017.

[9] S. Nagpal, M. Singh, M. Vatsa, and R. Singh, “Regularizing deep learning architecture for face recognition with weight variations," in Proc. Biometrics, Theory, Appl. Syst., 2015, pp. 1-6.

[10] W. Zhao, R. Chellappa, P. J. Phillips, and A. Rosenfeld, "Face recognition: A literature survey," ACM Comput. Surv., vol. 35, no. 4, pp. 399-458, Dec. 2003.

[11] Y. Sun, Y. Chen, X. Wang, and X. Tang, "Deep learning face representation by joint identication-verification," in Proc. Adv. Neural Inf. Process. Syst., vol. 27. 2014, pp. 1981-9196.

[12] M.-C. Hwang, L. T. Ha, N.-H. Kim, C.-S. Park, and S.-J. Ko, "Person identification system for future digital TV with intelligence," IEEE Trans. Consumer Electron., vol. 53, no. 1, pp. 218-226 ,Feb. 2007.

[13] Y. Adini, Y. Moses, and S. Ullma. Face recognition: the problem of compensating for changes in illumination direction. IEEE Transactions on Pattern Analysis and Machine Intelligence, 19:721-732, 1997.

[14] B. Jun and D. Kim, "Robust face detection using local gradient patterns and evidence accumulation," J. Pattern Recognition, vol. 45, no. 9, pp. 3304-3316, Sep. 2012.

[15] A. R. Rivera, R. Castillo, and O. Chae, "Local directional number pattern for face analysis: face and expression recognition," IEEE Trans. Image Process., vol. 22, no. 5, pp. 1740-1752, May 2013
[16] L. Lei, S.-W. Kim, W.-J. Park, D.-H. Kim, and S.-J. Ko, "Eigen directional bit-planes for robust face recognition," IEEE Trans Consumer Electron., vol. 60, no. 4, pp. 702-709, Nov. 2014.

[17] L. Lei, S.-W. Kim, W.-J. Park, D.-H. Kim, and S.-J. Ko, "Eigen directional bit-planes for robust face recognition," IEEE Trans Consumer Electron., vol. 60, no. 4, pp. 702-709, Nov. 2014.

[18] X. M. Turk and A. Pentland, "Eigenfaces for recognition," J. Cognitive Neuroscience, vol. 3, no. 1, pp. 71-86, 1991.

[19] G. Hua et al., "Introduction to the special section on realworld face recognition," IEEE Trans. Pattern Anal. Mach. Intell., vol. 33, no. 10, pp. 19211924, Oct. 2011.

[20] L. Best-Rowden, H. Han, C. Otto, B. F. Klare, and A. K. Jain, "Unconstrained face recognition: Identifying a person of interest from a media collection," IEEE Trans. Inf. Forensics Security, vol. 9, no. 12, pp. 2144-2157, Dec. 2014.

[21] Y. Huang, Z. Wu, L. Wang, and T. Tan, "Feature coding in image classification: A comprehensive study," IEEE Trans. Pattern Anal. Mach. Intell., vol. 36, no. 3, pp. 493506, Mar. 2014.

[22] S. Prabhakar, S. Pankanti, and A. K. Jain, "Biometric recognition: Security and privacy concerns," IEEE Security Privacy Mag., vol. 1, no. 2, pp. 33-42, 2003. 